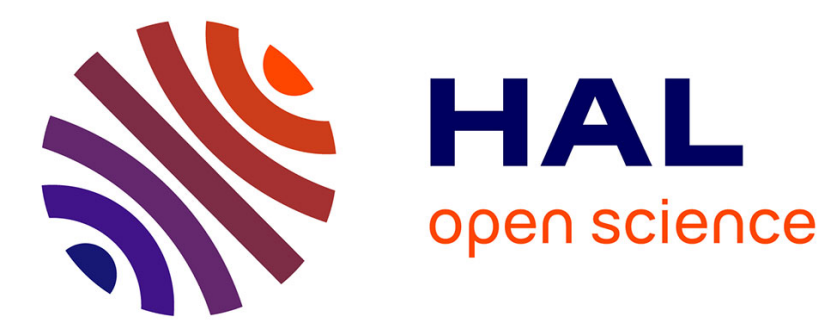

\title{
On Focal Length Calibration from Two Views
}

\author{
Peter Sturm
}

\section{To cite this version:}

Peter Sturm. On Focal Length Calibration from Two Views. IEEE International Conference on Computer Vision and Pattern Recognition (CVPR '01), Dec 2001, Kauai, United States. pp.145-150, 10.1109/CVPR.2001.990940 . inria-00525660

\section{HAL Id: inria-00525660 https://hal.inria.fr/inria-00525660}

Submitted on 26 May 2011

HAL is a multi-disciplinary open access archive for the deposit and dissemination of scientific research documents, whether they are published or not. The documents may come from teaching and research institutions in France or abroad, or from public or private research centers.
L'archive ouverte pluridisciplinaire HAL, est destinée au dépôt et à la diffusion de documents scientifiques de niveau recherche, publiés ou non, émanant des établissements d'enseignement et de recherche français ou étrangers, des laboratoires publics ou privés. 


\title{
On Focal Length Calibration from Two Views*
}

\author{
Peter Sturm \\ INRIA Rhône-Alpes \\ 655 Avenue de l'Europe, 38330 Montbonnot, France \\ Peter.Sturm@inrialpes.fr
}

\begin{abstract}
We consider the problem of self-calibration of a camera whose intrinsic parameters are known, besides the focal length. In the past, algorithms were mainly proposed for estimating two values of the focal length, for two images taken with different zoom. While there exist closed form solutions based on the fundamental matrix, their applicability is limited due to a generic singularity that occurs whenever the optical axes intersect, leading to numerical instabilities in most practical situations. Here, we consider the case of two views with identical focal length. We derive closed form solutions (one quadratic and two linear equations). Their respective singularity conditions are studied analytically and experimental results on their stability are given.
\end{abstract}

\section{Introduction}

It has been shown that camera self-calibration is possible by making rather simple assumptions on the intrinsic parameters. For example, the knowledge that pixels are rectangular allows in principle to (self-) calibrate all parameters of the pinhole model, even if they are different for all views $[9,13]$. In practice however, one usually tries to use as much a priori information on the intrinsic parameters as possible. Typically, it is possible to know the aspect ratio rather accurately, pixels are nearly perfectly rectangular and the assumption of the principal point in the center of the image has been shown to be valid [1]. Thus, it is possible to concentrate, in an initial stage, on the parameter most likely to be unknown, the focal length. If required, the other parameters may be refined subsequently, using bundle adjustment, which usually also includes parameters that model deviations from the perfect pinhole assumption.

Hartley has shown that the values of a varying focal length can already be estimated from two views of an unknown (static) scene [5]. Simple algorithms for this purpose are proposed in $[1,7,12]$. Basically, given the fundamental matrix of the two views, one can derive a quadratic equation

\footnotetext{
${ }^{*}$ This work was supported by the project IST-1999-10756, VISIRE.
}

in any of the two values of the focal length. The main drawback is the existence of singularities, especially the case of coplanar optical axes, which does not allow to compute the focal length, with any algorithm [12].

Brooks et al. have considered the case of identical focal length for the two views $[2,3]$. Their analysis is restricted to the case of a typical stereo system: the optical axes are perfectly coplanar and the relative motion between the two views is planar motion, the normal of the motion plane being parallel to the image plane's $x$ or $y$ axis. This gives rise to a special form of the fundamental matrix and a linear equation on the focal length. It has been noted that the coplanarity of the optical axes does not generally imply singularity, however singularity exists if the two vergence angles are identical (up to sign), or, equivalently, if the optical centers are equidistant from the optical axes' intersection point (including the case of parallel optical axes) [2].

In this paper, we generalize this work to any two-view configuration. We show that even in the general case, linear equations exist (on the squared focal length). Concretely, two linear and one quadratic equations can be derived from the singular value decomposition (SVD) of the fundamental matrix. We derive all generic singularities for the problem at hand, i.e. singularities that can not be solved by any algorithm. While the three equations are of course dependent in general, each one may be singular for different configurations. We reveal all such configurations, and especially show that the quadratic equation only suffers from generic singularities, whereas the linear equations are singular for coplanar optical axes and in another, less relevant case.

The paper is organized as follows. We analytically formulate the problem in $\S 2$. In $\S 3$, we derive the equations on the focal length. Generic and equation specific singularities are described in $\S \S 4$ and 5 respectively. The complete algorithm is given in $\S 6$. Simulation results are reported in $\S 7$, followed by conclusions in $\S 8$.

Notations. Geometric entities are represented by homogeneous coordinates. Matrices are represented in sans serif (e.g. K), vectors in bold face (e.g. q) and scalars in italics. The $i$ th coefficient of the vector $\mathbf{u}_{1}$ e.g., is written as $u_{1 i}$. 
Equality of matrices or vectors, up to scale, is denoted by $\sim$. For any vector $\mathbf{v},[\mathbf{v}]_{\times}$represents the skew-symmetric matrix associated with the cross product, i.e.: $\mathbf{v} \times \mathbf{w}=[\mathbf{v}]_{\times} \mathbf{w}$. Transposition of a vector $\mathbf{v}$ is noted as $\mathbf{v}^{\top}$ and the inverse of the transpose of a matrix $\mathrm{K}$ as $\mathrm{K}^{-\mathrm{T}}$.

\section{Problem Formulation}

Throughout this paper, we use perspective projection as camera model, with the following intrinsic parameters: the focal length $f$, the aspect ratio $\tau$ and the principal point $\left(u_{0}, v_{0}\right)$. A $3 \mathrm{D}$ point $\mathbf{Q}$ is projected to an image point $\mathbf{q}$ via:

$$
\mathbf{q} \sim \mathrm{PQ} \sim \mathrm{KR}(\mathrm{I} \mid-\mathbf{t}) \mathbf{Q}
$$

where the rotation matrix $R$ and the vector $t$ represent the camera's orientation and position, and the calibration matrix $\mathrm{K}$ is defined as:

$$
\mathrm{K}=\left(\begin{array}{ccc}
\tau f & 0 & u_{0} \\
0 & f & v_{0} \\
0 & 0 & 1
\end{array}\right)
$$

In the following, we suppose that two images of a static scene are given and that a projective reconstruction is possible or, equivalently, that the fundamental matrix can be computed. Without loss of generality, we assume that the first camera is located at the origin and that its rotation matrix is the identity. With $\mathrm{R}$ and $\mathbf{t}$ the extrinsic and $\mathrm{K}^{\prime}$ the intrinsic parameters of the second camera, the fundamental matrix of two images is given by [8]:

$$
\mathrm{F} \sim \mathrm{K}^{\prime-\mathrm{T}} \mathrm{R}[\mathrm{t}]_{\times} \mathrm{K}^{-1}
$$

We suppose that the aspect ratio and the principal point are known for both images and that their focal lengths are identical. We can thus move from a completely uncalibrated space to a "semi-calibrated" one, by computing an intermediate between the fundamental matrix and the essential ma$\operatorname{trix}\left(R[t]_{\times}\right.$in the above equation):

$$
\begin{aligned}
\mathrm{G} & \sim\left(\begin{array}{ccc}
\tau^{\prime} & 0 & 0 \\
0 & 1 & 0 \\
u_{0}^{\prime} & v_{0}^{\prime} & 1
\end{array}\right) \mathrm{F}\left(\begin{array}{ccc}
\tau & 0 & u_{0} \\
0 & 1 & v_{0} \\
0 & 0 & 1
\end{array}\right) \\
& \sim \operatorname{diag}(1,1, f) \mathrm{R}[\mathbf{t}]_{\times} \operatorname{diag}(1,1, f) .
\end{aligned}
$$

In the next section, we will establish equations for the computation of the focal length $f$, based on the singular value decomposition of the semi-calibrated fundamental matrix G.

Note that the optical axes are coplanar exactly if $G_{33}=0$ in the above parameterization ${ }^{1}$.

\footnotetext{
${ }^{1}$ This follows from the fact that coplanarity of the optical axes is equivalent to the principal points being in epipolar correspondence, and that the latter have coordinates $(0,0,1)$ in the semi-calibrated image spaces.
}

\section{Calibration Equations}

Let the singular value decomposition [4] of $\mathrm{G}$ be given by:

$$
\mathrm{G}=\mathrm{U} \Sigma \mathrm{V}^{\top}
$$

with $\Sigma=\operatorname{diag}(a, b, 0)$ the diagonal matrix of singular values $(a, b>0)$ and $\mathrm{U}$ and $\mathrm{V}$ orthogonal matrices. Let us denote by $\mathbf{u}_{i}$ and $\mathbf{v}_{j}$ the $i$ th respectively $j$ th column of $U$ respectively $V$. Note that the second epipole $\mathbf{e}^{\prime}$ associated with $G$ (i.e. the epipole of the semi-calibrated image plane) is its left nullvector and is thus equal (up to scale) to the third column of $\mathrm{U}$, i.e. $\mathbf{e}^{\prime} \sim \mathbf{u}_{3}$.

It can be shown [17] that the following relationship exists that links the fundamental matrix and the epipole, with the unknown intrinsic parameter (these equations are often called Kruppa equations):

$$
\mathrm{G} \operatorname{diag}\left(f^{2}, f^{2}, 1\right) \mathbf{G}^{\top} \sim\left[\mathbf{e}^{\prime}\right]_{\times} \operatorname{diag}\left(f^{2}, f^{2}, 1\right) \quad\left[\mathbf{e}^{\prime}\right]_{\times} .
$$

In terms of the SVD of $\mathrm{G}$, this can be written as:

$$
\begin{aligned}
U \Sigma \mathrm{V}^{\top} & \operatorname{diag}\left(f^{2}, f^{2}, 1\right) \vee \Sigma \mathrm{U}^{\top} \\
& \sim\left[\mathbf{u}_{3}\right]_{\times} \operatorname{diag}\left(f^{2}, f^{2}, 1\right)\left[\mathbf{u}_{3}\right]_{\times} .
\end{aligned}
$$

Multiplying the equation by $\mathrm{U}^{\top}$ from the left and $\mathrm{U}$ from the right gives, due to the orthogonality of $\mathrm{U}$ :

$$
\begin{aligned}
\Sigma \mathrm{V}^{\top} & \operatorname{diag}\left(f^{2}, f^{2}, 1\right) \vee \Sigma \\
& \sim \mathrm{U}^{\top}\left[\mathbf{u}_{3}\right]_{\times} \operatorname{diag}\left(f^{2}, f^{2}, 1\right)\left[\mathbf{u}_{3}\right]_{\times} \mathrm{U} \\
& \sim\left(\begin{array}{c}
\mathbf{u}_{2}^{\top} \\
-\mathbf{u}_{1}^{\top} \\
\mathbf{0}^{\top}
\end{array}\right) \operatorname{diag}\left(f^{2}, f^{2}, 1\right)\left(\begin{array}{lll}
\mathbf{u}_{2} & -\mathbf{u}_{1} & \mathbf{0}
\end{array}\right) .
\end{aligned}
$$

The last row and the last column of this matrix equation are zero vectors, so we concentrate on the upper left $2 \times 2$ part of the equation:

$$
\begin{aligned}
&\left(\begin{array}{c}
a \mathbf{v}_{1}^{\top} \\
b \mathbf{v}_{2}^{\top}
\end{array}\right) \operatorname{diag}\left(f^{2}, f^{2}, 1\right)\left(\begin{array}{ll}
a \mathbf{v}_{1} & b \mathbf{v}_{2}
\end{array}\right) \\
& \sim\left(\begin{array}{c}
\mathbf{u}_{2}^{\top} \\
-\mathbf{u}_{1}^{\top}
\end{array}\right) \operatorname{diag}\left(f^{2}, f^{2}, 1\right)\left(\begin{array}{ll}
\mathbf{u}_{2} & -\mathbf{u}_{1}
\end{array}\right) .
\end{aligned}
$$

Making use of the fact that the vectors $\mathbf{v}_{1}$ etc. have unit norm, we can further simplify:

$$
\begin{aligned}
& \left(\begin{array}{cc}
a^{2}\left(v_{13}^{2}\left(1-f^{2}\right)+f^{2}\right) & a b\left(v_{13} v_{23}\left(1-f^{2}\right)\right) \\
a b\left(v_{13} v_{23}\left(1-f^{2}\right)\right) & b^{2}\left(v_{23}^{2}\left(1-f^{2}\right)+f^{2}\right)
\end{array}\right) \\
& \sim\left(\begin{array}{cc}
u_{23}^{2}\left(1-f^{2}\right)+f^{2} & -u_{13} u_{23}\left(1-f^{2}\right) \\
-u_{13} u_{23}\left(1-f^{2}\right) & u_{13}^{2}\left(1-f^{2}\right)+f^{2}
\end{array}\right)
\end{aligned}
$$

The equality up to scale of these two symmetric matrices gives rise to three individual quadratic equations in $f^{2}$. Two 


$$
\begin{aligned}
f^{2}\left(a u_{13} u_{23}\left(1-v_{13}^{2}\right)+b v_{13} v_{23}\left(1-u_{23}^{2}\right)\right)+u_{23} v_{13}\left(a u_{13} v_{13}+b u_{23} v_{23}\right) & =0 \\
f^{2}\left(a v_{13} v_{23}\left(1-u_{13}^{2}\right)+b u_{13} u_{23}\left(1-v_{23}^{2}\right)\right)+u_{13} v_{23}\left(a u_{13} v_{13}+b u_{23} v_{23}\right) & =0 \\
f^{4}\left(a^{2}\left(1-u_{13}^{2}\right)\left(1-v_{13}^{2}\right)-b^{2}\left(1-u_{23}^{2}\right)\left(1-v_{23}^{2}\right)\right) & \\
+f^{2}\left(a^{2}\left(u_{13}^{2}+v_{13}^{2}-2 u_{13}^{2} v_{13}^{2}\right)-b^{2}\left(u_{23}^{2}+v_{23}^{2}-2 u_{23}^{2} v_{23}^{2}\right)\right)+\left(a^{2} u_{13}^{2} v_{13}^{2}-b^{2} u_{23}^{2} v_{23}^{2}\right) & =0
\end{aligned}
$$

of them have the trivial solution $f^{2}=1$. Factoring this out, we thus obtain two linear equations and a quadratic one, shown at the top of the following page. These are our (self) calibration equations. They are of course algebraically dependent, but we see in $\S 5$ that they may be singular in different conditions.

\section{Generic Singularities}

Before discussing singularities associated with the above calibration equations, we describe the generic singularities of the underlying problem, i.e. those that can not be overcome by any algorithm. Due to lack of space, we do not provide proofs for the following statements, but they can be obtained rather directly by specializing the results obtained for varying focal lengths [11, 12, 14, 15].

The only critical configurations for the (self) calibration of a constant focal length from two views occur if:

- the optical axes are parallel to each other.

- the optical axes intersect in a finite point and the optical centers are equidistant from this point.

Kahl and Triggs have derived critical configurations in [10]. However, their results are not as clearly stated as above, and it seems to us that they are slightly incomplete (their "turntable" rotation about the intersection point of the optical axes, can not produce all possible cyclotorsions of the two cameras).

In both critical cases, there are infinitely many solutions for the focal length.

Coplanarity of the optical axes is a necessary condition for a singular configuration with equal focal lengths, whereas it is already sufficient if two different focal lengths have to be estimated $[11,12,14,15]$. The different focal lengths case is subject to additional singularities (but which are not that likely to occur in practice). These are not generically singular for the equal focal length case, although we will see in $\S 5$ that they affect the two linear equations.

Intuitively, the stability of calibration in near-degenerate situations should be higher for the equal focal length case.

\section{Singularities of the Calibration Equations}

Naturally, it is worthwhile and even recommended to examine the singularities of the above calibration equations. They are indeed versions of the Kruppa equations which are known to suffer from additional singularities besides the generic ones $[17,16]$. We would thus like to determine under which conditions each of the equations becomes singular. This would allow to conclude if they suffer from nongeneric singularities and possibly to determine which equation to use under which condition, or to determine a single equation that should always be used.

Let us define singularity of the equations. All three calibration equations have the true squared focal length as solution. Singularity means the existence of additional, wrong solutions (however, we neglect here the spurious solution of the quadratic equation). This happens exactly if all coefficients of an equation vanish (there will be infinitely many solutions).

In the following, we establish the conditions where this is the case. Concretely, we determine singular relative camera poses. Due to lack of space, we omit all proofs; they can be found in an extended version of this paper ${ }^{2}$. The analysis is rather tricky, since the equations are formulated using the singular value decomposition of the fundamental matrix, which is not analytically available. Let us just note that the proofs are based on the fact that the free parameters (focal length, relative rotation $\mathrm{R}$ and relative position, up to scale, $t$ ) defining our matrix $G$, can not produce all possible $3 \times 3$ rank-2 singular value decompositions.

Naturally, all three equations vanish in the generic singular conditions given in $\S 4$. As for the quadratic equation, there are no further singularities (unlike the general Kruppa equations that are subject to non-generic singularities). As for the linear ones, they vanish as soon as the optical axes are coplanar, and in another, less relevant case that is illustrated in figure 1 . In the case of coplanar optical axes, the quadratic equation turns into a linear one, i.e. there is no spurious solution. In the other case where the linear equations vanish, the spurious solution of the quadratic equation for the squared focal length is always negative and can thus be ruled out. Hence, with the exception of generically singular configurations, it is always possible to have a

\footnotetext{
${ }^{2}$ http://www.inrialpes.fr/movi/people/Sturm
} 
unique solution (the true one if there is no noise): either the quadratic equation has a single admissible solution (positive value for squared focal length), or the spurious solution can be ruled out using the linear equations.

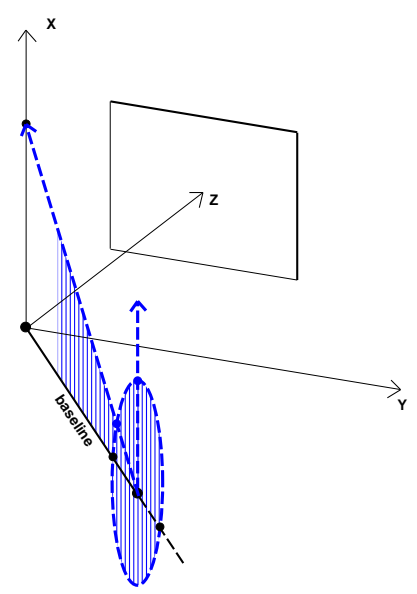

Figure 1. Example of a singular case for the linear equations when the optical axes are not coplanar. The configuration is singular for all directions for the second optical axis along the circle (two examples are shown).

As a sidenote, it is interesting to note that the singularities for the linear equations correspond to generically singular camera configurations for the case of different focal lengths (cf. $[11,12,14,15])$.

\section{Algorithm}

Our complete algorithm looks as follows:

1. Estimate the fundamental matrix between the two views (we use the linear method given in [6]).

2. "Undo" the known intrinsic parameters, as shown in equation (1).

3. Also undo a typical value $f_{0}$ for the focal length (usually of the order of $10^{3}$ ):

$$
\mathrm{G}^{\prime} \sim \operatorname{diag}\left(f_{0}, f_{0}, 1\right) \quad \mathrm{G} \operatorname{diag}\left(f_{0}, f_{0}, 1\right) .
$$

Further, scale $G^{\prime}$ e.g. to unit Frobenius norm. These two steps may improve numerical conditioning of the calibration equations, and we indeed observed a higher accuracy in practice.

4. Compute the SVD of $\mathrm{G}^{\prime}$ and extract the coefficients $u_{13}, u_{23}, v_{13}$ and $v_{23}$, as well as the non-zero singular values $a$ and $b$.
5. Construct and solve any of the equations (3) - (5). Multiply the solution(s) by $f_{0}$, to undo the effect of step 3 . In practice, we only solve the quadratic equation. The spurious solution can either be ruled out using the linear equations, or by simply taking the solution closest to $f_{0}$ (in simulations, the spurious solution was always observed to be far off the true one).

6. Optionally, the result can be improved by bundle adjustment, after having estimated the relative pose of the cameras.

\section{Experimental Results}

We conducted simulated experiments in order to assess the sensitivity of the calibration equations in close-tosingular situations. Figure 2 shows the simulated scenarios. The starting position of the cameras is depicted on the left: it is the typical stereo situation, with symmetric vergence angles. This situation is singular of course: the optical axes are coplanar and the optical centers are equidistant from the intersection point of the optical axes.
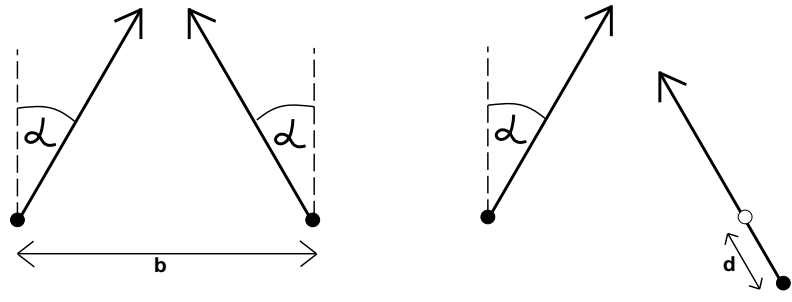

Figure 2. Simulation scenarios. Shown are the optical centers and optical axes. See text for more description.

In the first scenario, the second camera rotates away from the plane spanned by the initial position of the optical axes, by an angle between $0^{\circ}$ and $5^{\circ}$ ("elevation angle"). In figure 2 , this rotation would be towards the reader.

In the second scenario (shown on the right of figure 2), the second camera moves along its optical axis. The optical axes stay coplanar, but the distances of the optical centers to the intersection point of the optical axes are no longer equal. Hence, the scenario is not singular any more (generically, and for the quadratic equation), besides for the case of a zero vergence angle (parallel optical axes). The baseline of the system is $b=1000$ units, and the displacement of the second camera is by $d=-250,-200, \ldots, 250$ units.

For both scenarios, experiments are done for different vergence angles, with $\alpha$ between $0^{\circ}$ (parallel optical axes in the initial position) and $30^{\circ} .3 \mathrm{D}$ points in the common field of view are created randomly (each camera has a field of view of $25^{\circ}$ ). They are projected to the images and centered Gaussian noise with a standard deviation between 0 and 1 
pixels, is added to the image point coordinates. These image points are the input to the algorithm.

The following figures show results for the quadratic equation. Results for the linear equations are not shown here: they are singular in the second scenario, and rather unstable in the first one. Displayed are the median values of the relative errors on the focal length (the true value is 1000 , expressed in pixels), over 100 trials for each noise level.

First scenario: off-plane rotation. Figures 3 and 4 show results for this scenario, figure 3 relative to a zero vergence angle (i.e. with an elevation angle of $0^{\circ}$, the optical axes are parallel and the configuration is singular), and figure 4 relative to a vergence angle of $5^{\circ}$. For zero vergence, it can be seen that even for a $2^{\circ}$ rotation off the base plane, the errors are below $10 \%$ for realistic noise levels. Slight vergence of the cameras significantly improves the results errors are roughly halved (compare figure 4 with figure 3 ).

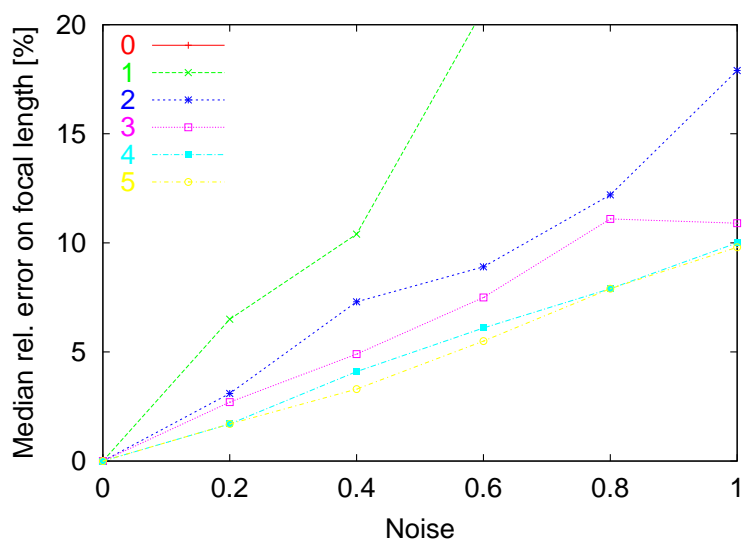

Figure 3. First scenario, varying elevation angle, fixed vergence: $\alpha=0^{\circ}$. The curve for $0^{\circ}$ elevation is outside the graph (this situation is singular, and the results reflect this).

Second scenario: displacement of the second camera. Figures $5-8$ show results for the second scenario. Figure 5 shows the influence of the vergence angle for a fixed, relatively small displacement ( $5 \%$ of the baseline) of the second camera. For a vergence angle of $0^{\circ}$, the optical axes are parallel and the situation remains singular for any displacement, which is reflected by the fact that the corresponding curve is outside the graph. Figure 5 shows that close to $0^{\circ}$ vergence, the results are still affected by the near-singularity and noise, but they stabilize with increasing vergence angle.

Figure 6 shows the failure rates for the same situation, for a noise level of 0.6. The algorithm was considered to have failed if the quadratic equation gave complex conjugate solutions. As expected, the failure rate is high for zero vergence and around $10 \%$ otherwise.

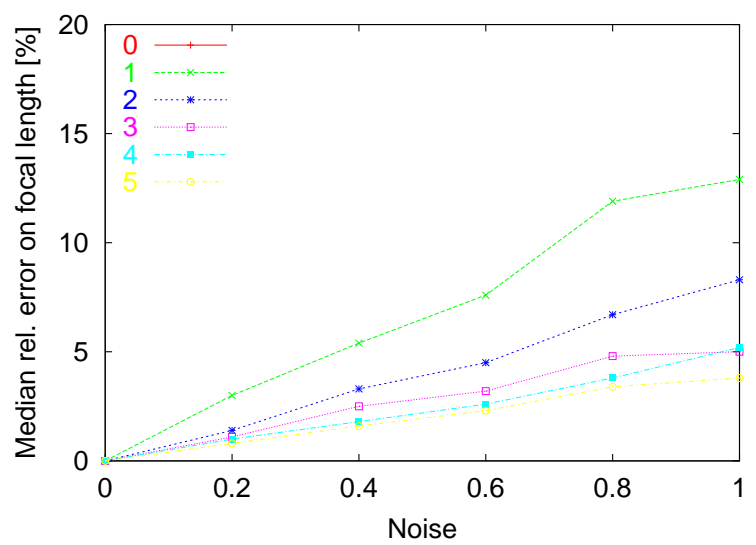

Figure 4. First scenario, varying elevation angle, fixed vergence: $\alpha=5^{\circ}$. The curve for $0^{\circ}$ elevation is outside the graph (singularity).

Figure 7 shows the results with respect to varying displacement, for a fixed vergence angle. The curve for zero displacement is outside the graph, the others show a roughly symmetric behaviour: lowest are the curves for the largest displacements, of \pm 250 , and highest the curves for the small displacements of \pm 50 units. Figure 8 shows that the failure rates get rather low with increasing displacement of the second camera.

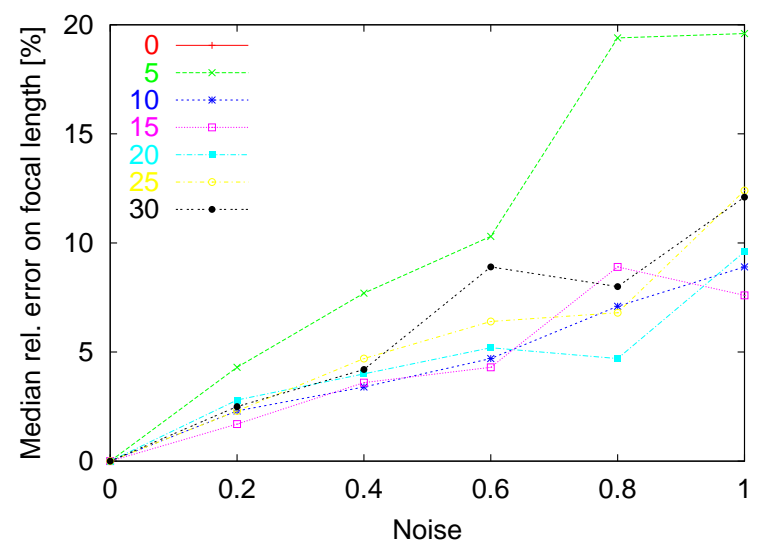

Figure 5. Second scenario, varying vergence angle, fixed displacement: $d=-50$ units.

\section{Conclusions}

We have analyzed the problem of focal length calibration from two views of an unknown scene, given their epipolar geometry and the assumption that the views have identical focal length. Closed form solutions have been derived, which consist in one quadratic and two linear equations 


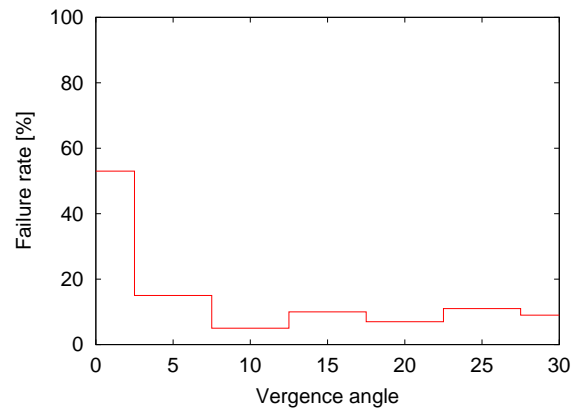

Figure 6. Same situation as in figure 5. Shown is the failure rate for a noise level of 0.6 .

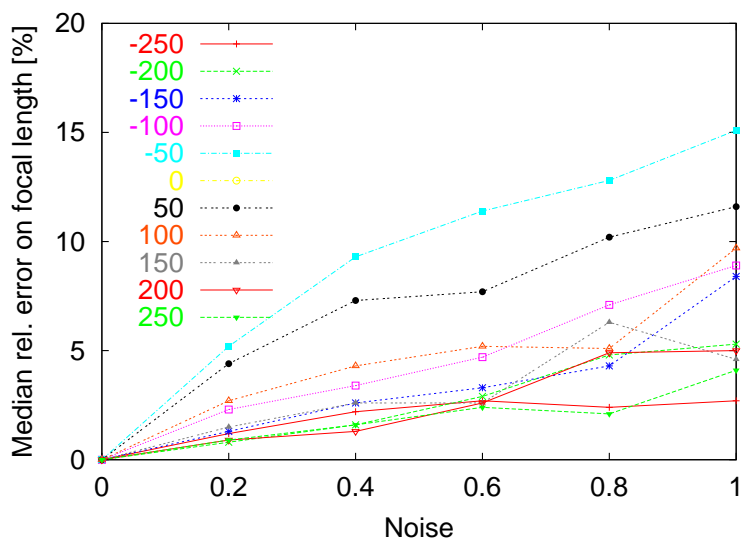

Figure 7. Second scenario, varying displacement, fixed vergence: $\alpha=10^{\circ}$.

(which are algebraically dependent). We have described the camera configurations that are generically singular for the calibration problem at hand and have derived all additional configurations in which one or more of the calibration equations vanishes. The analysis has shown that the quadratic calibration equation only vanishes in the generically singular configurations, namely if the optical axes are parallel or if the optical centers are equidistant from the (finite) intersection point of the optical axes. While in practice one will often be close to such a situation, it is rather easier to avoid than the general situation of coplanar optical axes, in which case methods for estimating two different focal lengths, fail.

Simulated experiments have been conducted that seem to indicate that even relatively small deviations from singular configurations allow accurate and reliable calibration. (Accuracy is here interpreted relative to typical sub-optimal self-calibration methods.) Overall we believe that the proposed algorithm, together with the singularity analysis, may constitute a small, though useful module in systems for e.g. 3D modeling from images.

Future work will concern the evaluation of the algo-

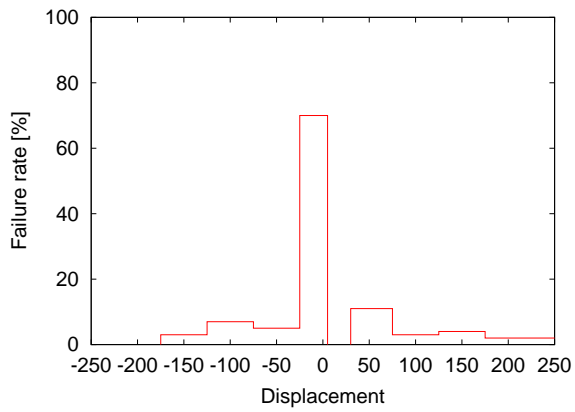

Figure 8. Same situation as in figure 7. Shown is the failure rate for a noise level of 0.6 .

rithm's sensitivity to errors in the assumed or given values of the other intrinsic parameters, as in [3], or to non perfectly identical focal lengths. It would also be interesting to derive a similar method for the three-view case, via the trifocal tensor.

\section{References}

[1] S. Bougnoux. From Projective to Euclidean Space under Any Practical Situation, A Criticism of Self-Calibration. ICCV, 790-796, 1998.

[2] M.J. Brooks, L. De Agapito, D.Q. Huynh and L. Baumela. Towards Robust Metric Reconstruction Via A Dynamic Uncalibrated Stereo Head. Image and Vision Computing, 16(14):989-1002, 1998.

[3] L. De Agapito, D.Q. Huynh and M.J. Brooks. Self-Calibrating a Stereo Head: An Error Analysis in The Neighbourhood of Degenerate Configurations. ICCV, 747-753, 1998.

[4] G.H. Golub and C.F. van Loan. Matrix Computation. The Johns Hopkins University Press, 1989.

[5] R.I. Hartley. Estimation of Relative Camera Positions for Uncalibrated Cameras. ECCV, 579-587, 1992.

[6] R. Hartley. In Defence of the 8-Point Algorithm. ICCV, 10641070, 1995.

[7] R.I. Hartley. Kruppa's Equations Derived from the Fundamental Matrix. IEEe Transactions on Pattern Analysis and Machine Intelligence, 19(2):133-135, 1997.

[8] R.I. Hartley and A. Zisserman. Multiple View Geometry in Computer Vision. Cambridge University Press, 2000.

[9] A. Heyden and K. Åström. Minimal Conditions on Intrinsic Parameters for Euclidean Reconstruction. ACCV, II:169-176, 1998.

[10] F. Kahl and B. Triggs. Critical Motions in Euclidean Structure from Motion. CVPR, 2:366-372, 1999.

[11] F. Kahl, B. Triggs and K. Åström. Critical Motions for AutoCalibration When Some Intrinsic Parameters Can Vary. Jrnl. of Mathematical Imaging and Vision, 13(2):131-146, 2000.

[12] G.N. Newsam, D.Q. Huynh, M.J. Brooks and H.P. Pan. Recovering Unknown Focal Lengths in Self-Calibration: An Essentially Linear Algorithm and Degenerate Configurations. ISPRS-Congress, XXXI(B3):575-580, 1996. 
[13] M. Pollefeys, R. Koch and L. Van Gool. Self-Calibration and Metric Reconstruction in Spite of Varying and Unknown Internal Camera Parameters. ICCV, 90-95, 1998.

[14] M. Pollefeys. Self-Calibration and Metric 3D Reconstruction from Uncalibrated Image Sequences. $\mathrm{PhD}$ Thesis, Katholieke Universiteit Leuven, Belgium, 1999.

[15] P. Sturm. Critical Motion Sequences for the Self-Calibration of Cameras and Stereo Systems with Variable Focal Length. BMVC, 63-72, 1999.

[16] P. Sturm. A Case Against Kruppa's Equations for Camera Self-Calibration. IEEE Transactions on Pattern Analysis and Machine Intelligence, 22(10):1199-1204, 2000.

[17] C. Zeller and O. Faugeras. Camera Self-Calibration from Video Sequences: the Kruppa Equations Revisited. Rapport de Recherche, No. 2793, INRIA, France, 1996. 